\title{
Better data with SEC-SAXS
}

Thomas M Weiss, Tsutomu Matsui, Ivan Rajkovic, Ping Liu

Stanford University, SSRL, Menlo Park, California

Size exclusion chromatography coupled to synchrotron SAXS data collection (SECSAXS) is a powerful tool for problematic biological samples such as weakly bound complexes, highly aggregation prone proteins or other complex mixtures of species that are not stable in their separated form. As such the technique has been implemented in one way or another at most synchrotron SAXS beam lines specializing in biological small angle solution scattering. The technique however is not fool proof and the data quality of the SEC-SAXS data can easily be compromised by radiation damage, capillary fouling and low signal to noise due to the dilution of the sample during the chromatography run. Here we will present how the optimized hardware implementation at SSRL BL4-2 as well as careful experimental planning can be used to address these problems and often eliminate them during data collection. 